\title{
Frequency Conversion With Positive Nonlinear Resistors
}

\author{
Chester H. Page
}

\begin{abstract}
A nonlinear resistor subject to an almost periodic voltage will absorb power at some frequencies, and supply power at other frequencies. Necessary and sufficient relations among these powers are found. Among the practical consequences are the results: (1) modulation efficiency cannot exceed unity, (2) subharmonics are not produced, and (3) the efficiency of generating an $n$th harmonic cannot exceed $1 / n^{2}$.
\end{abstract}

\section{Introduction}

Positive nonlinear resistors are here defined as two-terminal devices through which the current $(I)$ is a real finite single-valued nondecreasing function of the voltage $(V)$ across the terminals, with the added condition that $I(0)=0$. The function $I(V)$ may have simple discontinuities.

The voltage is assumed to be uniformly almost periodic, i. e., a bounded continuous function of time representable by a uniformly convergent trigonometric series.

The resistor will absorb power, $P_{n}$ (positive or negative), at each of the frequencies $\left(\omega_{n}\right)$ in the voltage. Relations among the $P_{n}$ are of practical and theoretical interest.

The voltage can be thought of as supplied by a series-connected set of generators, some of which are real, whereas others represent the effects of voltage drop across the remaining passive elements of the network. Generators associated with positive $P_{n}$ are called "sources"; those associated with negative $P_{n}$, "sinks." Continuity of $V(t)$ is provided pbysically by the inevitable shunt capacitance, however small, of the resistor.

$$
V \equiv \sum_{1}^{\infty} V_{n}=\sum_{1}^{\infty} a_{n} \cos \left(\omega_{n} t+\theta_{n}\right)
$$

The current through the resistor will be $S^{2}$ almost periodic, ${ }^{1}$ as is shown in the appendix. It is given almost everywhere by

$$
I(t)=\text { l. i. m. } \sum_{1}^{\infty} b_{n} \cos \left(\omega_{n} t+\varphi_{n}\right) .
$$

The frequencies $\omega_{n}$ may include some not required for the representation of $V$; these are included in eq (1) with vanishing coefficients. We denote the average power absorbed by the resistor at frequency $\omega_{n}$ by

$$
P_{n}=\left\langle V_{n} I[V(t)]\right\rangle_{t} \equiv \lim _{T \rightarrow \infty} \frac{1}{2 T} \int_{-T}^{T} V_{n}(t) I(t) d t
$$

\section{Modulation}

Partition the total voltage into two parts, a "carrier" $\left(V_{1}\right)$ and the combination of signal and modulation products, such that

$$
\begin{gathered}
V=V_{1}+V_{2} \\
\left\langle V_{2} I\left[V_{1}\right]\right\rangle=0
\end{gathered}
$$

defining as modulation products all frequencies (other than the signal) appearing in $V$ but not in the current that obtains in the absence of signal.

${ }^{1}$ Representability by eq (2) is essentially what is meant by Stepanoff almost periodicity. See A. S. Besicovitch, Almost periodic functions, ch. II, sections 2 and 3 (Dover Publications, New York, N. Y., 1954). 
Theorem. The sources in $V_{2}$ supply at least as much power as is absorbed by the sinks in $V_{2}$.

The total power supplied by the sources and sinks of $V_{2}$ is

$$
\left\langle V_{2} I\left[V_{1}+V_{2}\right]\right\rangle_{t}=\left\langle V_{2}\left\{I\left[V_{1}+V_{2}\right]-I\left[V_{1}\right]\right\}\right\rangle_{t}+\left\langle V_{2} I\left[V_{1}\right]\right\rangle_{t} \geq 0 .
$$

The first term on the right is nonnegative by the nondecreasing property of $I$, and the second term vanishes by the definition of $V_{2}$.

The total power available in all modulation products is therefore not greater than that supplied by the signal source, or modulation efficiency cannot exceed unity.

\section{Necessary Relations Among $P_{n}$}

The total power absorbed by the resistor is

$$
P=\langle V I\rangle_{t} \geq 0
$$

as $V I$ is a nonnegative function of $t$. Now

$$
0 \leq P=\langle V I\rangle_{t}=\left\langle\Sigma V_{n} I\right\rangle_{t}=\Sigma\left\langle V_{n} I\right\rangle_{t}=\Sigma P_{n}
$$

by virtue of uniform convergence. Further,

$$
P_{n}=\left\langle a_{n} \cos \left(\omega_{n} t+\theta_{n}\right) \Sigma b_{m} \cos \left(\omega_{m} t+\phi_{m}\right)\right\rangle_{t}=\frac{1}{2} a_{n} b_{n} \cos \left(\phi_{n}-\theta_{n}\right)
$$

so that

$$
\left|P_{n}\right| \leq \frac{1}{2}\left|a_{n} b_{n}\right|
$$

making

$$
\Sigma\left|P_{n}\right| \leq \frac{1}{2} \Sigma\left|a_{n} b_{n}\right| \leq \sqrt{\left(\frac{1}{2} \Sigma a_{n}^{2}\right)\left(\frac{1}{2} \Sigma b_{n}^{2}\right)}=\sqrt{\left\langle V^{2}\right\rangle\left\langle I^{2}\right\rangle}<\infty
$$

using Schwarz's inequality and the boundedness of $V$ and $I$. Similarly

$$
\Sigma P_{n}^{2} \leq \frac{1}{4} \Sigma a_{n}^{2} b_{n}^{2} \leq \frac{1}{4}\left(\Sigma\left|a_{n} b_{n}\right|\right)^{2} \leq \frac{1}{4} \Sigma a_{n}^{2} \Sigma b_{n}^{2}<\infty .
$$

The final condition follows from the nondecreasing behavior of $I$ :

$$
\theta(x) \equiv\langle\{\boldsymbol{V}(t)-V(t-x)\}\{I[V(t)]-I[V(t-x)]\}\rangle_{t} \geq 0 .
$$

Now

$$
\begin{aligned}
V(t)-V(t-x)=\Sigma a_{n}\left\{\cos \left(\omega_{n} t+\theta_{n}\right)-\cos \left(\omega_{n} t-\omega_{n} x+\theta_{n}\right)\right\} \\
\\
=\Sigma a_{n}\left\{\left[1-\cos \omega_{n} x\right] \cos \left(\omega_{n} t+\theta_{n}\right)-\sin \omega_{n} x \sin \left(\omega_{n} t+\theta_{n}\right)\right\}
\end{aligned}
$$

with a similar expression for $I(t)-I(t-x)$, making

$$
\begin{aligned}
\theta(x) & =\Sigma \frac{1}{2} a_{n} b_{n} \cos \left(\phi_{n}-\theta_{n}\right)\left\{\left(1-\cos \omega_{n} x\right)^{2}-\sin ^{2} \omega_{n} x\right\} \\
& =2 \Sigma P_{n}\left(1-\cos \omega_{n} x\right) .
\end{aligned}
$$

These results can be collected as the four conditions:
(a) $\Sigma P_{n} \geq 0$
(b) $\quad \Sigma\left|P_{n}\right|<\infty$
(c) $\Sigma P_{n}^{2}<\infty$
(d) $\quad \Sigma P_{n}\left(1-\cos \omega_{n} x\right) \geq 0$ all $x$.

\section{Sufficiency}

The above necessary conditions are also sufficient; given a set of $\omega_{n}$ and $P_{n}$ satisfying $(a)-(d)$, there exists at least one combination of allowed voltage and resistor producing a set of $P_{n}$ arbitrarily close to the specified values. 
For consider the voltage

$$
V=A^{2} \Sigma P_{n} \cos \omega_{n} t
$$

Condition (c) guarantees the Parseval equation and (b) makes $V$ uniformly almost periodic. Condition (d) shows that $V$ never exceeds its value at $t=0\left(V_{0}=A^{2} \Sigma P_{n}\right)$ and condition (a) makes $V_{0}$ positive. Let $V$ be applied across the resistor described by

$$
I=\left\{\begin{array}{l}
0, V \leq V_{0}-\delta \\
1, V>V_{0}-\delta
\end{array}\right.
$$

where $\delta$ is a small positive constant. There is a theorem ${ }^{2}$ that $V$ possesses translation numbers such that

$$
\left|\omega_{n} \tau\right|<\epsilon(\bmod 2 \pi) \quad \text { for } n<N \text {; }
$$

hence a relatively dense set of time intervals for which $V_{0}-\delta<V<V_{0}$, yielding unit current. By setting $1 / A^{2}$ equal to the fraction of the time that the current is unity, the power at $\omega_{n}$ is given by

$$
P_{n}^{\prime}=P_{n} \int_{I=1} \cos \omega_{n} t d t / \int_{I=1} d t
$$

that is, $P_{n}^{\prime} / P_{n}$ is equal to the average value of $\cos \omega_{n} t$ on the time intervals of unit current. This ratio can be made as close to unity as desired for any number $(N)$ of frequencies, by reducing $\delta$, for the continuity of $V$ in eq (11) provides a continuous reduction of the intervals of integration. For $n>N$, the arithmetic error $P_{n}^{\prime}-P_{n}$ can be made arbitrarily small by a sufficiently large choice of $N$, because by eq (14), $\left|P_{n}^{\prime} / P_{n}\right| \leq 1$, and $\Sigma\left|P_{n}\right|$ converges.

\section{Practical Theorems}

Three practical theorems can be deduced directly from the necessary and sufficient relations among the $P_{n}$. For the modulation theorem, we consider a sinusoidal carrier and signal, so that all $\omega_{n}$ are of the form $n \omega_{c}+m \omega_{s},(-\infty<n, m<\infty)$. Condition (d) yields

$$
\Sigma \Sigma P_{n m}\left[1-\cos \left(n \omega_{c}+m \omega_{s}\right) x\right] \geq 0 .
$$

For $x=2 \pi k / \omega_{c}, k$ an integer, this becomes

$$
\Sigma \Sigma P_{n m}\left[1-\cos 2 \pi m k \omega_{s} / \omega_{c}\right] \geq 0
$$

and we can choose $k$ to make the cosine less than $\epsilon$ for $m<M$ excepting the values $m=l \omega_{c} / \omega_{s}$, for which the cosine becomes unity. Therefore $\Sigma \Sigma P_{n m} \geq 0$, summing over all frequencies that are not harmonics of the carrier.

The second theorem states that subharmonics cannot be generated. Let $\tau$ be the least common period of the sources (or in general the least translation number of the source voltage). Then $P_{n}\left(1-\cos \omega_{n} \tau\right)$ vanishes for all positive $P_{n}$, and condition (d) requires this expression to vanish for each of the negative $P_{n}$. Hence all sinks have the period $\tau$; all generated frequencies are harmonics of difference frequencies.

The third theorem limits the efficiency of harmonic generation to $1 / n^{2}$. For

making

$$
P_{1}(1-\cos \omega x)-\left|P_{n}\right|(1-\cos n \omega x) \geq 0
$$

$$
\frac{\left|P_{n}\right|}{P_{1}} \leq \frac{1-\cos \omega x}{1-\cos n \omega x}=\frac{\sin ^{2} \omega x / 2}{\sin ^{2} n \omega x / 2}
$$

\footnotetext{
${ }^{2}$ A. S. Besicovitch, Almost periodic functions, p. 53 (Dover Publications, New York, N. Y., 1954).
} 
As $x \rightarrow 0$, the ratio of sines squared goes to its minimum value of $1 / n^{2}$.

The positive definite form (d) can be generalized:

Theorem. If $g(\omega)=\omega \int_{0}^{\infty} \varphi(x) \sin \omega x d x$, where $\varphi(x)$ is a nonincreasing positive function, and the integral is summed in the Cesàro sense, then $J[g] \equiv \Sigma g\left(\omega_{n}\right) P_{n}$ is positive definite.

For

$$
\begin{aligned}
g(\omega) & =\lim _{\lambda \rightarrow \infty} \int_{0}^{\lambda}(1-x / \lambda) \varphi(x) d(1-\cos \omega x) \\
& =\lim _{\lambda \rightarrow \infty}\left\{\frac{1}{\lambda} \int_{0}^{\lambda} \varphi(x)(1-\cos \omega x) d x+\int_{0}^{\lambda}(1-x / \lambda)(1-\cos \omega x)(-d \varphi)\right\}
\end{aligned}
$$

upon integrating by parts. Then

$$
J[g]=\lim \frac{1}{\lambda} \int_{0}^{\lambda} \varphi(x) \Sigma P_{n}\left(1-\cos \omega_{n} x\right) d x+\lim \int_{0}^{\lambda}(1-x / \lambda) \Sigma P_{n}\left(1-\cos \omega_{n} x\right)(-d \varphi)
$$

using the uniform convergence of $(d)$. By $(d)$ and the restrictions on $\varphi$, there are no negative terms on the right, so

$$
J[g] \geq 0 .
$$

Corollary:

$$
J(\alpha) \equiv \Sigma \omega_{n}^{\alpha} P_{n} \geq 0 \quad \text { for } 0 \leq \alpha<2 .
$$

In this range of $\alpha, J$ vanishes only for the trivial case of all $P_{n}$ zero. The closure $J(2)$ is positive semidefinite; it vanishes for $V=\sin ^{3} \omega t, I(V)=0$ for $V \leq 0, I(V)=1$ for $V>0$.

\section{Appendix}

Let $I(V)$ have only a finite number of simple discontinuities in any finite range of $V$. Let these be at the points $V_{i}$. Let $0<\delta<$ g.l.b. $\left|V_{i}-V_{j}\right|$ and let l.u.b. $|I(V+\delta)-I(V)|=B$ so that for any $0<\epsilon<\delta,|I(V+\epsilon)-I(V)| \leq B$. For $\left|V-V_{i}\right|>\delta, I$ is continuous in the interval $[V, V \pm \epsilon]$, and $\epsilon$ can be chosen to make $|I(V \pm \epsilon)-I(V)|<\zeta$, where $\zeta$ is any assigned positive number. Therefore,

$$
|I(V \pm \boldsymbol{\epsilon})-I(V)| \leq\left\{\begin{array}{l}
\zeta,\left|V-V_{i}\right|>\delta \\
B,\left|V-V_{i}\right| \leq \delta
\end{array}\right.
$$

Now if $V(t)$ is uniformly almost periodic, but not periodic, we can choose $\delta$ so that on the interval $t_{0} \leq t \leq t_{0}+l$, the measure of $t$ for which $\left|V-V_{i}\right| \leq \delta$ is less than $l \zeta^{2}$. Then

and

$$
\frac{1}{l} \int_{t_{o}}^{t_{0}+l} \mid I\left[V(t) \pm \epsilon_{-}^{\prime}-\left.I[V(t)]\right|^{2} d t \leq B^{2} \zeta^{2}+\zeta^{2}\right.
$$

$$
\underset{-\infty<t_{o}<\infty}{\text { l.u.b. }}\left\{\frac{1}{l} \int_{s_{0}}^{t_{o}+l}|I[V(t) \pm \epsilon]-I[V(t)]|^{2} d t\right\}^{1 / 2}<\zeta \sqrt{B^{2}+1}
$$

Now the translation numbers $\tau$ of $V(t)$ make $|V(t+\tau)-V(t)|<\epsilon$ so that

$$
\text { l.u.b. }\left\{\frac{1}{l} \int_{0}^{t_{0}+l}|I[V(t+\tau)]-I[V(t)]|^{2} d t\right\}^{1 / 2}<\zeta \sqrt{B^{2}+1}
$$

and $I(t)$ is Stepanoff almost periodic, with a Fourier Series which converges to $I(t)$ in the mean square.

If $V(t)$ is periodic, then $I(t)$ is also per odic, hence Stepanoff a. p. a fortiori.

Washington, Decembei 19, 1955. 